\title{
Filigrane
}

Écoutes psychanalytiques

\section{L'avenir de la psychanalyse : un nouvel humanisme ? Entretien avec Roland Gori}

\section{Laurence Branchereau}

Volume 23, numéro 2, automne 2014

URI : https://id.erudit.org/iderudit/1028927ar

DOI : https://doi.org/10.7202/1028927ar

Aller au sommaire du numéro

Éditeur(s)

Revue Santé mentale au Québec

ISSN

1192-1412 (imprimé)

1911-4656 (numérique)

Découvrir la revue

Citer ce document

Branchereau, L. (2014). L'avenir de la psychanalyse : un nouvel humanisme ? Entretien avec Roland Gori. Filigrane, 23(2), 127-144.

https://doi.org/10.7202/1028927ar d'utilisation que vous pouvez consulter en ligne. 


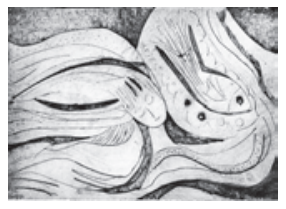

\section{L'avenir de la psychanalyse: un nouvel humanisme? Entretien avec Roland Gori ${ }^{1}$}

\section{Laurence Branchereau}

Laurence Branchereau : Alors, il me fait plaisir Roland Gori de vous accueillir aujourd'hui pour échanger avec vous sur votre parcours et sur les réflexions que vous développez depuis plus de 10 ans, à propos de l'esprit du temps qui façonne notre manière de voir et d'appréhender le monde. C'est votre premier séjour à Montréal, je crois, et pour ceux qui ne vous connaîtraient pas, vous êtes psychanalyste, professeur émérite de psychopathologie clinique à l'Université Aix-Marseille, et vous avez écrit de nombreux ouvrages dont tout récemment La fabrique des imposteurs. Vous avez aussi publié en 2011 La dignité de penser, et avec d'autres collègues, La folie évaluation. En 2008, avec Marie-José Del Volgo, vous publiiez Exilés de l'intime. La médecine et la psychiatrie au service du nouvel ordre économique et en 2005, La santé totalitaire. Essai sur la médicalisation de l'existence. Vous exposez dans ces différents ouvrages des thèses pour nous aider à comprendre ce qui se passe dans nos sociétés actuelles concernant le rapport à soi et à l'autre, et l'incidence des transformations sociales et culturelles. Pourriez-vous nous parler des grands axes de vos réflexions et aussi de l'intérêt que vous avez eu, comme psychanalyste, à vous prononcer sur ces questions?

Roland Gori: Je vous remercie de votre invitation, Laurence Branchereau, et de cette possibilité d'exposer quelques-uns de mes thèmes privilégiés. Peut-être rappeler un peu mon parcours. Je me suis toujours intéressé à la question de la parole et du langage. J'évoque cette question parce que, d'une certaine manière, le langage implique toujours quelque part le politique. Qu'on le sache ou non, implicitement, il y a une dimension politique selon la conception que l'on a du langage. Je me suis d'abord intéressé à l'acte de parole dans une perspective presque scientiste, c'est-à-dire une analyse en «cluster» du matériel verbal de groupes thérapeutiques, en essayant de mettre en relation des indices linguistiques et des processus psychologiques. 
Et puis, assez rapidement, je me suis aperçu de l'impasse, au moins relative, que peut constituer ce type de recherche, et je me suis davantage intéressé à la dimension de l'acte de parole comme le lieu d'investissement des pulsions sexuelles partielles par exemple, mais aussi comme le lieu de constitution des idéaux narcissiques. Il s'agissait d'une approche psychanalytique de l'acte de parole. J'ai fait d'autres recherches du côté de l'hystérie, du côté de la logique des passions. Et puis, en France notamment mais aussi au-delà, je me suis rendu compte que la psychanalyse, qui avait été portée pendant quelque temps et notamment durant la décennie des années 1960-1970 par la culture, subissait un relatif discrédit dans l'opinion publique. Ce discrédit de la psychanalyse en tant que mode de connaissance et pratique thérapeutique était-il dû à des événements scientifiques majeurs qui justifiaient qu'on change de référentiel, qu'on change de paradigme aussi bien dans nos pratiques cliniques que dans nos modes de recherche théorique? Ou est-ce qu'il s'agissait d'autre chose?

Pour un temps, je me suis davantage intéressé à la question de la validité de ce qu'en psychanalyse on pouvait avancer comme données de connaissance et comme pratiques cliniques. Je me suis ainsi intéressé à ce qu'on pourrait appeler l'épistémologie de la psychanalyse et de la psychopathologie clinique, c'est-à-dire à quelle condition un énoncé théorique référé à la psychanalyse peut s'avérer pertinent ou alors, relever d'une espèce d'idéologie, puisque la culture a absorbé comme une éponge quelques énoncés psychanalytiques sur la fonction du père, sur le complexe oedipien, sur la sexualité. On a vu récemment d'ailleurs en France, à l'occasion de la loi sur «le mariage pour tous», que certains psychanalystes tentaient de transformer la psychanalyse en guide des bonnes mœurs, pour le meilleur et pour le pire. En d'autres termes, je me suis intéressé aux conditions qui permettaient de considérer qu'une pratique, un travail ou un savoir psychanalytique soit valide et fiable. Je suis arrivé à la conclusion que la psychanalyse n'est pas un vocabulaire, un savoir verbal, ni même conceptuel ou notionnel; ce n'est pas seulement une situation divan-fauteuil. La psychanalyse est d'abord et avant tout la mise en ouvre d'une méthode dans une pratique clinique utilisant l'efficience symbolique du langage. Pour le dire autrement, on ne peut savoir grand-chose des symptômes au nom desquels les patients viennent nous consulter tant qu'ils ne sont pas rapatriés dans le cadre d'une situation avec laquelle ils vont faire corps. Il n'y a pas d'interprétation des symptômes tant que ceux-ci ne passent pas par leur actualisation au cœur de la situation analytique au sein de laquelle se réalise leur déchiffrage à partir 
de leur adresse transférentielle. C'est un point qui pour moi est important, notamment en lien avec les travaux du docteur Conrad Stein. Il faut d'abord et avant tout que les processus psychologiques soient insérés dans le cadre d'une séance de psychanalyse, conçue d'ailleurs comme un rêve parlé, pour que l'on puisse en exhumer la signification subjective.

Cela réduit considérablement le champ de la psychanalyse. Et à partir de ce moment, la psychanalyse peut être considérée comme un laboratoire avec une situation quasi-expérimentale, avec une méthode, avec des données qui sont d'ailleurs des artefacts d'une certaine manière. Le propre de l'objet scientifique est d'être produit par un dispositif phénoménotechnique. Quand Lavoisier dit «la chimie crée son objet», il montre bien que la chimie ne s'occupe pas de la nature en générale, mais des éléments de la nature qu'elle a pu rapatrier dans un cadre et qu'elle a recréés, décomposés, recomposés à sa façon. Il est important de considérer la psychanalyse un peu comme la chimie; c'est d'ailleurs une métaphore freudienne: la décomposition et la recomposition des processus psychopathologiques ou psychologiques dans le cadre d'une cure où la dimension transférentielle est essentielle. On ne peut rien dire d'un symptôme tant qu'il n'a pas été rapatrié dans une situation incluant l'analyste, qui autorise sa transformation en ce que Ferenczi appelait des symptômes transitoires, soit les symptômes qui apparaissent et disparaissent au cours d'une séance, au cours d'une cure analytique. C'est presque un principe méthodologique de considérer que tout ce qui va être vécu, apporté au cours de l'analyse, et pas seulement au cours de la séance, relève d'une adresse transférentielle. Ce point est pour moi très important parce qu'il insiste sur la dimension transférentielle de ce qui peut être interprété. De plus, l'interprétation n'est pas une interprétation de l'analyste donnant un sens à ces processus transférentiels mais plutôt, ce par quoi l'analyste amène l'analysant à conduire sa propre cure. Le bénéfice pour l'analyste est, comme le dit Stein d'ailleurs, de poursuivre sa propre analyse au-delà du temps des séances qu'il a eues avec son propre analyste.

Au départ, c'est plutôt dans le sens d'une purification de ce qui est constitutif de la spécificité de l'analyse et du travail de l'analyste qu'ont commencé mes recherches en ce domaine. Ce qui implique de se débarrasser d'une idéologisation psychanalytique du monde et de ce qui se passe dans le social et le culturel, d'où mes réserves concernant un certain nombre d'extrapolations psychologisantes des problèmes sociaux ou des problèmes culturels. Une fois ce travail accompli, il y a moyen de savoir ce qui relève véritablement d'une procédure de travail analytique comme méthode et ce 
qui se dilue du savoir analytique dans la culture, d'une idéologie analytique au sens de Canguilhem: cet état paresseux du savoir qui fait de l'idéologie psychanalytique un cas particulier de l'idéologie scientifique, c'est-à-dire une extrapolation de données partielles acquises au sein d'un laboratoire et qu'on va ensuite appliquer à l'ensemble du monde. Il existe un tas de situations similaires. Par exemple, même si des recherches très intéressantes démontrent le rôle de l'ocytocine dans le rapprochement des animaux, ce n'est pas pour autant qu'on va expliquer le lien social par l'ocytocine!! De la même manière, des études intéressantes concernant le différentiel entre la température du jour et de la nuit dans le cas de certaines populations de déprimés ne peuvent permettre de déduire que toute dépression découle d'un dysfonctionnement endocrinien. Il faut avoir ce principe épistémologique et éthique à l'esprit, et éviter de généraliser des données très partielles que l'on possède, que ce soit dans le champ des sciences pures, des sciences sociales, des sciences humaines, voire de la psychanalyse, pour expliquer le monde!

Arrivé à ce point, il est vrai que les psychanalystes pourraient avoir une certaine responsabilité dans le malheur qui leur arrive, et qu'ils ont pu vibrer aux appels de la culture, vibrer à l'intérêt social que leur pouvoir peut avoir eu, à une certaine époque, pour expliquer les relations d'autorité à l'aide de la psychologie et de la psychanalyse. C'est notre part dans le symptôme du discrédit de la psychanalyse, mais bon, c'est le cas aussi de beaucoup de champs de savoir, de pratiques. Je reviens donc à cette question: qu'est-ce qui fait qu'aujourd'hui la psychanalyse a mauvaise presse? Est-ce que des événements scientifiques majeurs peuvent expliquer cette situation? Selon moi, non. Il y a des découvertes scientifiques partielles qui justifient l'intérêt des recherches en neurobiologie du comportement, qui justifient et légitiment l'intérêt de recherches en épigénétique du comportement et dans les sciences cognitives. Je suis pour une biodiversité des théories et des pratiques, donc je suis pour une biodiversité épistémologique puisqu'elle me parait partie prenante de la biodiversité de la nature et de la culture.

Attardons-nous maintenant non plus sur l'épistémologie ou sur la validité des énoncés, mais analysons plutôt ce que Michel Foucault appelle la généalogie des savoirs, soit la fabrication de ceux-ci. Quel est l'impensé d'un savoir? Impensé cognitif ou conceptuel, impensé social aussi, puisque c'est l'apport de Michel Foucault par rapport à Canguilhem; Canguilhem et Foucault étant pour moi des références majeures. Considérons que l'accueil que des données théoriques ou des pratiques peuvent recevoir à une époque 
dans une société donnée ne dépend pas simplement de leur pertinence épistémologique, mais aussi de ce que Bourdieu a appelé leur utilité sociale. Qu'est-ce que le pouvoir peut en faire en tant que discours de légitimation sociale pour s'autoriser à donner les ordres?

J'ai travaillé notamment sur la façon dont les sciences du vivant, et la médecine en particulier, étaient depuis environ la fin du XviII ${ }^{e}$ siècle utilisées comme discours de légitimation sociale. À la suite de Foucault, j'ai considéré l'effacement des grands récits collectifs à la fin du XviII ${ }^{\mathrm{e}}$, le désenchantement du monde au sens de Marcel Mauss et au sens de Max Weber... ce désenchantement du monde, cette désacralisation des discours, autorisent à donner des ordres, font que le pouvoir se tourne de plus en plus vers les sciences du vivant et en particulier vers la médecine pour gérer les populations. Je ne vais pas développer, mais cela réfère à tout le travail de Foucault sur la biopolitique des populations, sur la biohistoire, sur la somatocratie, c'est-à-dire toutes les stratégies qui visent à dicter, au nom du bienêtre, au nom de la santé, la manière dont vous devez vous conduire. De ce fait, les sciences et les experts deviennent quasiment des sophistes. Ils vont vendre leur savoir pour justifier que le pouvoir puisse dire aux populations comment elles doivent se comporter pour bien se porter. On constate par exemple dans cette chose indigente que constituent les dernières versions du DSM, comment effectivement sont prescrites des normes de comportements. Les prochaines versions vont sûrement nous annoncer le nombre de fois où nous avons le droit d'avoir des rapports sexuels, avec qui, à partir de quel moment, si on est trop en-dessous ou trop au-delà... enfin bref, la manière dont on doit consommer, dont on doit devenir l'entrepreneur de notre existence. Nous nous dirigeons de plus en plus vers une espèce de calibrage des comportements comme on calibre les aliments. On va dire volontiers en Europe que Bruxelles n'ayant aucune autorité politique, la seule justification des commissions, qui coûtent extrêmement cher d'ailleurs, est de produire des règlements, du droit mou, des circulaires et autres visant à calibrer la taille des pommes, quitte à perdre la saveur même des fruits et des légumes. Et on tend à faire de même avec les humains! Cela n'empêche pas qu'il y ait une "politique Findus», comme je l'appelle, qui fait qu'on va suivre attentivement la trajectoire d'un produit, mesurer la dimension des étiquettes, voir si elles sont bien rédigées dans une ou deux langues, mais on ne va pas s'intéresser à ce qu'il y a dedans, ni au goût du produit, ni même à la vérité de sa substance. C'est pour cela d'ailleurs qu'on a retrouvé de la viande de cheval à la place de la viande de bœuf... dans le scandale français 
de l'entreprise de produits surgelés Findus!! Je plaisante à peine, parce que je pense que c'est ce que l'on retrouve aujourd'hui dans différents champs des sciences sociales et médicales.

Nous nous sommes intéressés, Marie-José Del Volgo et moi, à cette médicalisation de l'existence, à la façon dont la médecine, au-delà des énoncés scientifiques pertinents qu'elle peut apporter, peut être utilisée à des fins de pouvoir et pas seulement à des fins de connaissance ou de traitement. Il nous a semblé qu'il s'agissait de la fabrique d'une santé totalitaire. Bien sûr, nous ne subissons plus aujourd'hui des pouvoirs politiques totalitaires - comme nous en avons connus malheureusement au $\mathrm{xx}^{\mathrm{e}}$ siècle avec le stalinisme, le fascisme et le nazisme —, mais nous vivons néanmoins dans des formes culturelles totalitaires qui prescrivent de manière impérative la manière dont nous devons vivre, la manière dont nous devons exister.

Ce mythe d'une administration scientifique et technique du vivant a émergé au $\mathrm{XIX}^{\mathrm{e}}$ siècle, dans le cadre d'une philosophie, d'une sociologie positiviste qui prétendait se débarrasser du politique. L'idée était en quelque sorte de gouverner les hommes de façon neutre, purement technique et rationnelle, en se débarrassant de l'aspect politique; ce fut un thème extrêmement important dans la deuxième moitié du XIX ${ }^{e}$ siècle. En se débarrassant de la question du langage, on peut maintenant gérer l'humain en faisant du bouturage, de l'agriculture de l'humain; une culture de l'humain à partir des données de la science et de la technique. On va aboutir à une alimentation fourragère des résidents du grand âge lorsqu'on va déterminer la composition de leurs repas à partir de leur indice de graisse et de l'analyse de leurs selles! On perd le goût de la vie en l'administrant comme dans l'élevage et l'abattage! De telles mesures devraient rester exceptionnelles et temporaires, faute de quoi elles deviendraient monstrueusement totalitaires!

Ce point de vue très hygiéniste va participer, dans la deuxième moitié du XIX ${ }^{e}$ siècle, à la création d'une forme de psychiatrie très impliquée dans le social et à la construction d'un sujet psychologico-moral, inséré dans sa famille, sa sexualité, son histoire, ses affects. La psychanalyse participait en quelque sorte à ce mouvement, c'est incontestable. C'est dire que finalement, le social va demander à la psychiatrie de prédire comment le criminel ressemblait à son crime avant même de l'avoir commis.

Il est évident que la découverte pasteurienne est fondamentale dans cette promotion de l'hygiène, puisqu' elle résout une fois pour toute la question de l'étiologie des maladies en insistant sur la spécificité des agents pathogènes. Le développement des recherches en ce sens va aussi participer à la salubrité 
publique et l'emphase sur l'hygiène va permettre aux médecins de constater combien les conditions de travail participent à la santé ou aux maladies des populations, mais cette évolution va aussi soutenir une gestion presque technico-politique du social. Il ne faut pas croire que le savoir demeure sans conséquence sur le social et le politique. C'est un peu ce que je voudrais essayer de montrer. Cette idée d'une administration scientifique et technique des populations et des individus, ce rêve positiviste va devenir un cauchemar au $\mathrm{xx}^{\mathrm{e}}$ siècle puisque les totalitarismes politiques vont se prévaloir d'un idéal d'amélioration de l'espèce ou de sens de l'histoire pour accomplir leurs crimes. Les nazis vont dire "nous avons fait de la biologie appliquée», et le stalinisme va prétendre, au nom du matérialisme historique, orienter la tendance d'une civilisation ou culture, d'individus ou de peuples. Je crois que la psychanalyse, comme la psychothérapie institutionnelle, la phénoménologie et les humanités, a constitué une alternative à cette réduction de l'humain à sa dimension la plus animale et la plus économique. C'est pour ça que j'aime bien parler du concept anthropologique de l'homme neuroéconomique. Je crois que la psychanalyse, comme d'autres modes de savoir, a proposé une alternative à cette gestion et à cette conception purement technico-scientiste de l'humain.

Le problème est que, depuis trois ou quatre décennies, on voit très nettement apparaittre une nouvelle conception, que je trouve totalitaire, du monde. Pour aborder celle-ci - en faisant allusion aux ouvrages que vous avez bien voulu mentionner: La dignité de penser, La fabrique des imposteurs, et plus récemment Faut il renoncer à la liberté pour être heureux (2014) -, j'emprunterai l'analyse à un essayiste pour qui j'ai une très grande admiration, le cinéaste Pasolini qui parle de la religion du marché et nous montre comment la société bourgeoise n'a plus besoin des valeurs traditionnelles d'ordre, de progrès, de religion, de famille, et de nation, pour imposer une gouvernance qui permette une circulation maximale des échanges et la lutte tendancielle contre la baisse des taux de profit. Le marché est devenu une morale et une psychologie autant qu'un système économique et politique.

Comme le montre Pasolini, le marché s'est lui-même constitué comme religion monothéiste et presque intégriste dans cette conception du monde. Je ne vais pas développer davantage ce point, mais dans cette société de la marchandise et du spectacle, nous n'avons plus besoin des mêmes savoirs et pratiques. Il m’a semblé, par rapport à certaines recherches généalogiques que j'ai pu faire dans ce domaine, que la recomposition aujourd'hui de la place des savoirs, des humanités, ou du champ psychiatrique, nous 
fait apercevoir une nouvelle hiérarchie des savoirs et des pratiques avec une clinique qui compte de moins en moins. Dans l'étude des populations, le sujet n'est plus qu'un segment de population statistique, au détriment d'une analyse au cas par cas ou de la singularité clinique. Pour le dire très simplement, j'ai été amené à étudier un certain nombre de pièces maîtresses de ces dispositifs qui participaient au discrédit d'un certain mode de traitement de l'humain et de sa vulnérabilité. Vous savez ce que dit Jean Pic de la Mirandole lorsqu'il parle de ce qui fait la dignité de l'homme: c'est son état de néoténie, d'extrême faiblesse et de dépendance lorsqu'il naît, de même que sa longue enfance, qui le font dépendre de quelqu'un. Cette dépendance produit aussi bien la névrose que la culture.

Le mode de traitement actuel de la vulnérabilité, de la faiblesse, me parait refléter davantage les valeurs de notre époque que des découvertes techniques ou scientifiques majeures. Analysons l'apparition du DSM III, les modifications du DSM-IV, la préséance accordée aux thérapies cognitivo-comportementales parce que cette psychologie environnementale a une affinité avec une économie politique des comportements, au sein de laquelle l'humain est conçu comme un sujet qui doit résoudre des problèmes ou optimaliser ses compétences pour accroître ses performances. N’y a-t-il pas une affinité entre certaines manières de penser l'humain et de nommer son mal-être? Il n'y a pas que le traitement qui est concerné, même nos diagnostics reflètent ces valeurs. Par exemple, quelque chose qui me parait aussi mou que le diagnostic de dépression, fait fureur; comme si la dépression était un diagnostic qui s'imposait sur des critères observables très précis. Lorsque j'ai travaillé en psychiatrie au tout début de ma carrière, face à un état de très grande tristesse d'un patient, toute une analyse sémiologique après un entretien clinique était menée, pour savoir si cette dépression, cette tristesse était consécutive à des évènements de perte, réactionnelle à la perte d'un être cher, d'un idéal, d'un emploi, etc., ou si elle faisait partie d'un tableau névrotique, notamment dans l'hystérie où émergent des périodes un peu sombres, ou bien si on était face à une trajectoire maniaco-dépressive ou encore s'il s'agissait du tableau profond et sombre de la mélancolie. Bref, il y avait un travail, une finesse sémiologique qui aujourd'hui est complètement balayée. Qu'est-ce que la dépression? C'est ce que guérit un antidépresseur. Les maux de tête peuvent être liés à la dépression, de même que des douleurs physiques. Il peut y avoir dépression sans tristesse, et la dépression peut ne pas être reconnue comme telle.

Il me semble qu'on est dans une société qui, au nom du pragmatisme et de l'utilitarisme, méprise profondément le sujet dans sa singularité, dans 
son histoire et dans le sens qu'il cherche à sa vie. En 1980, lorsque l'homosexualité est supprimée de la liste des troubles des comportements sexuels, par ce simple geste administratif est abolie une inconsistance épistémologique et scientifique dans la manière même dont les diagnostics de troubles du comportement sexuel étaient posés. C'est pratique de parler de dépression. C'est pratique et c'est très poreux aussi par rapport aux exigences de la culture. Quand en France, entre 1979 et 1996 on multiplie par sept le nombre de diagnostics de dépression, ça ne veut pas dire qu'il y a sept fois plus de déprimés, ça signifie que ce diagnostic est posé sept fois plus souvent, et régulièrement par des généralistes plutôt que des psychiatres. Cela signifie aussi que la société est moins tolérante par rapport aux troubles émotionnels et de l'humeur, dont l'humeur dépressive. C'est un peu ce qu'on a essayé de démontrer dans Exilés de l'intime, et que j'ai poursuivi dans un autre livre, De quoi la psychanalyse est-elle le nom? Ouvrages dans lesquels j'ai tenté d'analyser les dispositifs de diagnostic et de traitement comme étant révélateurs des valeurs à l'usage dans la culture, avec cette idée que le savoir nait de la niche écologique d'une culture qu'il participe en retour à recoder.

Considérons les diagnostics de déprimé ou d'hyperactif: ce n'est pas d'une grande finesse quand même comme diagnostics, et la manière de les poser est plutôt rudimentaire! Selon moi, nous assistons à l'une des premières fois où une psychiatrie peut fonctionner sans psychopathologie. Pourquoi? Parce qu'elle est simplement devenue une hygiène publique du corps social. Elle n'a plus la guérison pour objectif, elle n'a plus la compréhension du patient comme souci premier, ni même, de lui permettre d'accroître son savoir sur lui-même et de s'épanouir davantage. L'objectif est plutôt de n'être pas trop déprimé, pas trop hyperactif. On règle les comportements comme on règle les rouages d'une machine. Les robots sont déjà parmi nous... Cette situation a quand même des conséquences sociales et culturelles graves, en fabriquant des enveloppes culturelles problématiques où le sujet se forme néanmoins, mais en perdant toujours davantage son humanité. Le diagnostic d'hyperactivité explose, mais ce diagnostic est-il scientifique? Je n'en suis pas du tout convaincu. J'en tiens pour exemple une étude suisse et allemande au cours de laquelle l'on a proposé à 1000 médecins et psychothérapeutes quatre cas d'enfants et d'adolescents un peu agités, dont un seul relevait vraiment du diagnostic strict de trouble de l'attention avec hyperactivité. Il y a eu surdiagnostic, imputable notamment au sexe du patient — les cliniciens surdiagnostiquent davantage les garçons que 
les filles - et au sexe du médecin ou du psychothérapeute: les cliniciens hommes ont tendance à surdiagnostiquer davantage que les femmes.

Cela illustre bien la très grande porosité du champ de la psychiatrie. On ne définit pas les normes psychiatriques de la même manière que les normes biologiques. En 1974, lorsque la ligue allemande de lutte contre l'hypertension artérielle a fait baisser d'un point la norme de la pression diastolique (le chiffre le plus bas) de la tension artérielle, le nombre de patients atteints d'hypertension s'est vu multiplié par trois... mais on ne peut pas vraiment descendre en-dessous. Du point de vue de la psychiatrie, il y a beaucoup plus de porosité; la classification diagnostique va inclure par exemple le trouble de l'humeur des femmes qui ont leurs règles en tant que dysphorie prémenstruelle. À partir du moment où vous constatez qu'il n'y a pas d'immaculée conception du savoir, de la pratique, des diagnostics et des traitements, en psychiatrie mais aussi dans d'autres champs de la connaissance, il vaut la peine de s'intéresser aux niches culturelles d'où émergent les vecteurs qui recomposent le champ des savoirs.

C'est à cela que je me suis intéressé avec notamment Marie-José Del Volgo, Jean-Claude Maleval, Alain Abelhauser et Marie-Jean Sauret, dans La folie évaluation. Nous nous sommes aperçus que les nouvelles formes d'évaluation sont des dispositifs de servitude volontaire et de soumission sociale librement consentie qui sont organisées selon une supposée objectivité - qui est souvent une objectivité purement formelle, de conformité, c'est-à-dire de procédures, ou une objectivité relative à des indices quantitatifs. Avec ce type d'évaluation, on espère calibrer les savoirs et les pratiques, ce que l'on fait de plus en plus au regard des exigences sociales et culturelles. À quoi servent la littérature, le psychodrame, la psychanalyse, la philosophie et l'histoire? Il est évident que les humanités sont perdantes, de même que la part d'humanité dans l'homme risque de se perdre. Nous sommes aujourd'hui face à une nouvelle forme de civilisation des mœurs qui bien sûr pousse en avant certaines formes de savoirs et de pratiques, mais empêche aussi d'autres formes de savoirs et de pratiques. Aujourd'hui, on préférera la neurobiologie ou la génétique du comportement, non pas parce que le politique ou le social aurait un amour fou pour les sciences, mais tout simplement parce qu'il est plus facile d'aller chercher l'hyperactivité de l'enfant dans tel ou tel gène que dans la surstimulation dont les enfants peuvent être l'objet aujourd'hui face aux écrans et aux nouvelles technologies, ou face à la déliaison du lien familial ou social. Il y a des formes de savoir qui disculpent la société de la part qui est la sienne dans la fabrique des symptômes, et 
c'est ce type de savoir qui va être favorisé aujourd'hui, d'où ce retour massif vers la génétique des comportements, qui par ailleurs est une science formidable. Mais je ne crois pas que ce soit par innocence politique et sociale qu'elle est favorisée. Du côté de la psychologie, ce sont plutôt les formes utilitaires, pragmatiques et techniques qui sont favorisées, non pas du point de vue de ce qu'elles apportent en termes de savoir, mais en tant que descriptions de comment les individus se comportent. Ce qu'ils sont dans leur comportement, c'est autre chose; c'était la grande thèse winnicottienne: où est le sujet dans ce qu'il fait, quels sont les investissements psychiques dans le comportement? Quelles sont les positions subjectives dans le comportement? On s'en fout complètement dans la psychiatrie d'aujourd'hui! À la limite, si vous avez un comportement normal, ou même si vous êtes éventuellement inadapté et que cette inadaptation est en quelque sorte une psychose blanche, ça ne gêne absolument personne. Ce qui compte c'est la manière dont vous vous comportez et la probabilité actuarielle d'avoir des comportements indésirables!

D'où le travail que j'essaie de faire ces derniers temps autour du fait qu'en favorisant les exigences de l'adaptation à des normes au détriment des conditions qui permettent la création et l'innovation, on fabrique aujourd'hui des imposteurs. Il n'y a pas mieux que l'imposteur pour savoir jouer des formes, des rituels, des codes, et s'adapter dans le semblant. C'est cette dimension-là qui m’a amené à m'intéresser toujours plus à cet environnement culturel qui est le nôtre. Je le fais modestement pour les citoyens, en tant que citoyen qui a eu par ailleurs une longue pratique universitaire avec de multiples responsabilités et activités, puis bien sûr en tant vieux praticien de la psychothérapie et de la psychanalyse qui peut attester de son expérience. Mais surtout pas en tant qu'expert. Je n'aime pas quand les journalistes me disent «qu'en pense le psychanalyste?». Mais le psychanalyste ne pense pas quoi que ce soit. Le psychanalyste travaille dans son fauteuil et de temps en temps il écrit des choses, mais il ne va pas avoir une pensée sur l'affaire Cahusac ou Zampino. C'est le citoyen qui va dire qu'est-ce qui fait qu'aujourd'hui il y a autant d'inégalités sociales et économiques dans certaines régions du monde. Est-ce que ça ne risque pas de menacer la démocratie? Est-ce que le citoyen a une question par rapport à ça, ou il n'y a plus cette passion de l'égalité qui a été à l'origine de pas mal de révolutions ou d'indépendance? Est-ce que ce n'est pas la démocratie elle-même qui est malade et qui fait qu'on voit émerger des inégalités sociales et économiques? Si aujourd'hui on voit apparaitre de plus en plus d'histoires de fraude, de tricherie, d'imposture, est-ce parce qu'on 
est dans un monde plus transparent, ou parce que dans le champ même du politique, la vertu n'est plus requise comme qualité pour gouverner?

J'essaie de montrer que l'imposteur est aujourd'hui comme un poisson dans l'eau parce que notre mode d'évaluation fait prévaloir la forme sur le fond, le semblant sur le vrai, la conformité des procédures sur la pertinence de leur acte eu égard aux finalités qui sont les leurs comme soigner, chercher, enseigner, juger, etc. J'essaie de montrer qu'il y a une coextensivité entre l'imposture et l'inflation des normes. Une part de mes travaux antérieurs insistaient sur cette extension sociale de la norme qui à mon avis révèle une crise de l'autorité: quand l'autorité est en crise, on développe des dispositifs normatifs qui font pression et qui constituent des modes d'emploi de l'existence. Il est évident que la psychanalyse, on peut en dire beaucoup de mal et on a bien raison de dire du mal de certains psychanalystes. Mais la psychanalyse a le mérite de constituer ce grain de sable qui vient gripper une machine qui fait de l'humain une pièce détachée de l'espèce, de la société, ou de l'économie. C'est très intéressant pour les traitements la médecine basée sur les preuves et la statistique des populations, mais il y a une dimension oubliée qui est le cas par cas. Le cas par cas c'est la clinique. Je crois que la psychanalyse possède cette qualité d'être ultra clinique.

J'ai tendance à penser que la psychanalyse n'a pas toujours existé et que rien ne garantit qu'elle puisse toujours exister. Je pense qu'elle a fait partie du retour à la fin du XIx ${ }^{\mathrm{e}}$ siècle d'un souci de soi, comme dit Foucault, d'une culture de soi, qui était propre à la culture antique et qui tendait à être recouverte au moment de cette industrialisation massive, de cette urbanisation massive du $\mathrm{XIX}^{\mathrm{e}}$ siècle, avec l'émergence de formes de savoir de plus en plus forte du côté de l'économie et de la biologie. Finalement, à la fin du XIX $^{\text {e }}$ siècle, la psychanalyse recueille ce qui ne fonctionne pas dans les modèles dominants de savoir, par exemple la biologie ou l'économie: un reste de l'homo economicus, un reste de l'homme biologique.

Il n'est pas anodin que ce soit les hystériques, qui mettent le fonctionnement du corps en panne, qui aient amené Freud à sa découverte - outre le génie freudien. Freud était tourné vers la culture classique et s'est trouvé happé par une modernité, voire une postmodernité, qu'il récusera. Il n’a jamais accueilli à bras ouverts d'être élu par les surréalistes par exemple; en témoigne l'échec de la rencontre avec Breton. Donc, à un moment donné, ça ne marche pas: ça ne marche pas dans le corps, ça ne marche pas au nom de l'économie, et ça ne marche pas non plus au niveau des savoirs modélisés sur le corps et sur l'économie. Il faut à la fois qu'il y ait cet environnement 
qui promeut ce type de savoir totalitaire où l'humain est en quelque sorte absorbé dans un dispositif qui ne traite de lui que le bioéconomique... et il faut qu'il y ait un reste. Les patients ont incarné ce reste, ce rebut des modèles neuroéconomiques de la fin du XIx ${ }^{e}$ siècle! Les hystériques ont été les symptômes de cette civilisation d'une certaine manière, elles ont été les emblèmes de cette résistance à la réduction de l'humain à un pur fonctionnement corporel ou aujourd'hui, à un pur fonctionnement de stratège économique.

Il est possible qu'on assiste à un retour du balancier à partir de ce système totalitaire, scientiste et économique qui financiarise l'humain et qui en même temps naturalise cette financiarisation de l'humain. Aujourd'hui, c'est l'époque du neuromarketing, de la neuroéconomie. Il y a une thèse qui a été publiée il y a quelques années sur la neuroéconomie ${ }^{2}$, qui vantait les métaphores croisées entre le marché et le fonctionnement du cerveau. Si le cerveau fonctionne comme un marché, le marché fonctionne naturellement comme un cerveau. Et en ce moment, il y a plutôt une commotion cérébrale du marché; j'espère que la morale et la psychologie que son système impose en seront un peu ébranlées, mais je n'en suis pas convaincu!

L'échec de ces savoirs-là et le désaveu que ce type d'approche recevrait éventuellement par l'expérience concrète, quotidienne et sociale pourraient amener un retour du balancier. Si ce n'est pas la psychanalyse, ce sera peutêtre une autre forme de culture de soi, une autre manière de prendre soin de soi; c'est l'avenir qui décidera. On ne peut pas entrer dans l'avenir à reculons en pensant qu'on va reproduire le passé. Cela signifie aussi que la psychanalyse n'est pas forcément tributaire des quatre à cinq séances par semaine; d'abord, les gens n'en ont plus les moyens. Une analyse ne dure pas forcément 20 ans, 30 ans ou toute la vie; et la psychanalyse n'est pas nécessairement incarnée par le divan et le fauteuil. Peut-être qu'un travail psychanalytique qui dépasse largement le cadre réduit d'une situation presque mobilière saura être reconnu dans sa pertinence. D’après mon expérience dans les services de médecine actuels (de génétique, de cancérologie, de soins palliatifs, psychiatriques, et de services pédiatriques), il y a cet appel à traiter le reste de l'approche biomédicale. Plus les cliniciens sont armés scientifiquement, plus ils ressentent la nécessité que quelqu'un d'autre s'occupe de ce qui reste. Cependant, moins l'approche est scientifique ou plus elle est indigente comme la psychiatrie, moins les cliniciens éprouvent ce besoin de traiter le reste. Voilà pourquoi je ne crois pas du tout à la néopsychiatrie très inspirée par les DSM et compagnie: c'est davantage d'hygiène sociale qu'il s'agit. 
Tout de même, il y a cette demande sociale qui est aussi une demande singulière. Auprès de quelqu'un qui apprend qu'il a un cancer, vous pouvez utiliser tous les dispositifs d'annonce imaginables... il n'empêche qu'il faudra en quelque sorte aider ce patient à élaborer ce qui lui arrive et accompagner sa prise en charge médicale. Ce patient va écrire, ce que nous avons appelé avec Marie-José Del Volgo un roman de sa maladie; il a besoin de donner du sens à ce qui lui arrive. Pour citer Canguilhem, la maladie, avant d'être la limitation d'une fonction physique, est d'abord et avant tout un drame de l'existence. Ce serait redoubler la souffrance et la cruauté que d'ignorer cette part, ce besoin de mettre en fiction ce qui nous arrive. Ce qui ne veut absolument pas dire que ce sens qu'il a besoin de donner à sa maladie, à son malheur ait le moindre rapport avec la cause de sa pathologie! À ce niveau, il ne faut pas opposer en médecine l'approche biomédicale et la prise en charge psychologique des patients: elles relèvent de deux régions ontologiques et méthodologiques différentes. On peut très bien diagnostiquer de l'asthme mais pour un patient, la crise d'asthme arrive à un moment donné et prend un certain sens. L'asthme construit de manière médicobiologique et l'asthme en tant qu'élément existentiel, signifiant qui surgit à un moment donné de la séance sur le divan, ne sont pas du même registre. Bref, à moins que la civilisation des mœurs parvienne à nous transformer en robot, en machine ou en animaux primitifs, il y aura nécessairement ce reste, et il appartient à la psychanalyse - mais pas seulement à la psychanalyse, à bien d'autres formes de soin, et de pensée aussi — de recueillir ce reste.

Par ailleurs, il y a aussi le devoir pour la psychanalyse de sortir de la morale d'épicier comme disait Marx, et d'aller se frotter aux exigences du social. Il faut arrêter de penser que la psychanalyse est persécutée, que les psychanalystes sont les héros incompris d'un temps cruel. À ce propos, j'aimerais dire quelques mots sur L'appel des appels.

J'ai eu la chance, à la suite de la parution de certains bouquins comme Les exilés de l'intime ou La santé totalitaire, d'aller rencontrer d'autres professionnels que les psychologues, psychiatres, psychanalystes. Je suis donc sorti un peu de ma communauté et ce qui m’a frappé, c'est que cette souffrance (je n'aime pas trop le terme) des praticiens aujourd'hui qui obtiennent difficilement les conditions requises pour exercer leur métier, je l'ai rencontrée chez les pédiatres, les dermatologues, les orthophonistes, les enseignants et chez les chercheurs - même les chercheurs en informatique et en mathématiques. Il y a eu aussi cette révolte des psychiatres et des psychologues, dont je faisais partie, contre certains propos concernant les schizophrènes 
dangereux, puis «Pas de zéro de conduite», un mouvement contre le dépistage de la délinquance à partir du trouble de conduite des enfants de trois ans, qui a abouti à des stratégies de réhabilitation sociale de type compétence émotionnelle et habileté sociale, hors de tout souci de sagesse ou de rapport à soi-même. Ainsi se fabriquent des individus qui sont des microentreprises libérales autogérées certes, ouvertes à la compétence certes, mais aussi en proie à la concurrence sur le marché existentiel. Cette conception entrepreneuriale du sujet humain, je n'y adhère pas volontiers, ni politiquement, ni au niveau de ma clinique singulière avec des patients.

Ce fut pour moi une découverte, et avec Stéfan Chedri, nous avons lancé L'appel des appels, ce collectif qui visait à alerter l'opinion et les pouvoirs publics sur le danger auquel s'exposaient toutes nos professions, par le biais de l'évaluation et de la recomposition de nos métiers au nom de la rentabilité, de la gestion, de la performance et de l'excellence, faisant perdre de vue la visée initiale pour laquelle elles avaient été créées. Un médecin est d'abord créé pour soigner un patient; il n'a pas à accroître la quantité d'argent qui entre dans son pôle médical, ce qu'on appelle en France la T2A: la tarification à l'activité. Si vous voulez incorporer dans le geste professionnel d'autres critères que les critères qui lui sont spécifiques - soigner, enseigner, chercher, juger, informer, etc. - cela pervertit l'acte professionnel. Aujourd'hui, par le biais de l'évaluation qui se veut quantitative, procédurale et formelle, on calibre de plus en plus les actes et finalement on introduit de plus en plus dans la tête des praticiens un souci de rentabilité, un souci de résultats à court terme. Notre civilisation du court terme me parait extrêmement dangereuse. De même, les évaluations scolaires ne testent pas le désir d'apprendre des élèves ni l'augmentation de leur savoir, elles testent simplement leurs réactions comportementales à certains stimuli. Robert Lind avait fait un travail très intéressant là-dessus, en sciences de l'éducation. Il avait montré que l'amélioration des résultats aux tests d'évaluation scolaire mesurait davantage l'entraînement des élèves à passer le test que le volume de connaissances qu'ils pouvaient s'approprier. Et cela se retrouve partout. Par exemple, chez les journalistes, ce qui compte, c'est le nombre de commentaires produits à partir de l'émission, le nombre de lecteurs qui vont s'intéresser à l'article, et pas du tout la qualité de l'article ou de l'émission. Cette logique de l'audimat constitue le cheval de Troie d'une logique du marché dans l'ensemble des champs professionnels, et opère par le biais de l'évaluation. Pour moi, la cible c'est vraiment l'évaluation. Non pas qu'il faille s'abstenir de rendre des comptes: il faut rendre des comptes, ça fait 
partie du devoir démocratique. Non pas qu'il faille s'abstenir de se comparer: la comparaison est nécessaire dans une démocratie où l'humain n'est pas assigné à un rang par sa naissance, à contrario de l'aristocratie. Mais il faut le faire en fonction de la spécificité de nos actes, et non pas du caractère conforme et formel de ces actes eu égard aux valeurs sociales en vogue à une certaine époque.

Cette situation conduit les gens à une solution de l'imposture. Qu'est-ce que c'est que l'imposture? C'est simplement le fait de vendre des apparences, du faire-semblant, pour essayer d'échapper aux contraintes normatives qui font vivre les individus ou les peuples au-dessus de leurs moyens. Avec Karl Abraham, Hélène Deutsch, Donald Winnicott, on comprend bien comment les individus, face à une intrusion de l'environnement, adoptent une solution qui vise à jouer les caméléons pour se défendre de l'environnement menaçant, intrusif, ou qui les néglige.

Donc, pour moi, voilà ce qu'est L'appel des appels. Et ce qui est fantastique, c'est que deux psychanalystes lancent cet appel qui dit simplement que nous ne sommes pas d'accord avec les réformes qui, au nom d'un ordre économique, transforment nos métiers en instruments dans le pouvoir politique, transforment l'homme en instrument. Et nous avons reçu un écho très fort. La presse en a parlé, nous avons été d'abord à la une de Libération; Le Monde aussi en a tenu compte, Médiapart, puis ensuite c'est surtout L'Humanité, Politis, France culture, France inter, France 2, France 3... qui ont suivi le soutien de Libération. Nous avons eu une très importante couverture médiatique, et aujourd'hui, nous avons plus de 90000 signatures du manifeste.

Ce mouvement s'est concrétisé, par exemple, par l'organisation de journées nationales: une première journée le 31 janvier 2009 au 104, qui est un centre culturel à Paris, où il y avait plus de 1200 personnes; en mars de la même année, à Montreuil (à la Maison de l'Art) où il y avait plus de 1200 personnes; à Marseille où il y avait plus de 1000 personnes. Donc, il y a des regroupements. On sent le besoin des gens de venir témoigner de leur expérience. C'est pour ça que je n'aime pas l'expression de «souffrance au travail» parce qu'elle me parait appartenir à une logique victimaire, issue d'une société où l'on ne peut faire reconnaître sa citoyenneté et sa subjectivité que par le biais de cette logique victimaire, en tant que «victime de». Pourtant, on sent le besoin de raconter son histoire, de témoigner: voilà ce qui se passe dans ma classe, voilà ce qui se passe aujourd'hui chez un psychologue, voilà ce qu'on me demande de faire... Qu'il s'agisse de jeunes 
psychiatres, cancérologues, infirmiers, journalistes, d'acteurs culturels, etc., ce besoin de témoigner et de partager est très, très fort.

Par la suite, nous avons créé un réseau. Ce mouvement a intéressé des Suisses, des Belges, des Italiens, et on espère un comité québécois de L'appel des appels. Nous ne sommes pas un parti politique, nous n'avons pas de sections. Nous ne disons pas aux gens comment ils doivent faire. Nous demandons seulement de signer une charte qui est une charte de partage de valeurs humanistes. Et après, chacun s'organise un peu. Certains ont arrêté, d'autres ont démarré tout petit et continuent quand même. Chacun s'auto-organise et on essaie d'échanger par les réseaux. Si internet peut être un dispositif qui confisque l'existence pour des fins de soumission, de marchandisation des données personnelles par exemple, le web est aussi un moyen formidable d'émancipation. Mediapart en France en est un bel exemple. Les textes, les vidéos, les interviews circulent, de même que les soutiens. Nous sommes quand même aujourd'hui à une époque où les grandes entités d'autorité, qui à la fois peuvent contraindre les individus mais aussi les soutenir, les étayer, sont mises à mal, et il nous faut trouver d'autres façons de vivre ensemble. Nous avons créé une association nationale avec un conseil de l'Appel des appels; nous organisons des journées de réflexion. La prochaine (le 25 mai 2013) sera intitulée «Est-ce qu’on peut encore exercer son métier de journaliste aujourd'hui?», avec des invités comme Stéphane Paoli de France-Inter, Edwy Plenel de Mediapart, Charles Sylvestre de L'Humanité, Sophie Peters de La Tribune et d'autres jeunes bien sûr. Nous avons organisé plusieurs journées sur l'évaluation, ce qui nous a amenés à demander la suppression des agences d'évaluation afin qu'elles soient remplacées de manière beaucoup plus pertinente par des conseils de professionnels ou de citoyens. Nous ne voulons pas revenir à une évaluation corporatiste entre professionnels, il faut des pairs bien sûr pour «juger» mais aussi des citoyens lambda. Les citoyens qui utilisent les services doivent aussi donner leur avis et ils peuvent aussi évaluer la façon dont ils sont soignés, reçus, enseignés, jugés, etc. Et il est nécessaire aussi d'y associer les financeurs publics et privés, of course!

Voilà ce qu'est devenu L'appel des appels.

Laurence Branchereau: Merci, Roland Gori, d'avoir accepté de nous entretenir sur vos récents écrits, vos réflexions des plus actuelles, et vos implications sociales. 


\section{Notes}

1. Cette entrevue - également disponible en ligne, sur notre site web - a été réalisée le 15 avril 2013, à Montréal. Roland Gori avait été invité par la Maison St-Jacques à l'occasion du colloque célébrant le 40ème anniversaire de cet organisme.

2. Gauthier, Martin (2006). Les neurosciences et l'économie: perspectives de la neuroéconomie. Mémoire de maîtrise, UQAM. 